\section{Betalaktame oft zu unrecht verdächtigt}

Bis zu 10\% der Patienten, bei denen eine Betalaktamantibiotikaallergie durch Haut- und Provokationstests ausgeschlossen wurde, reagieren trotzdem auf diese Substanzgruppe - so war bisher die gängige Meinung. Die Gefahr scheint aber wesentlich geringer zu sein, wie eine aktuelle prospektive Studie zeigt.

든 in französisch-schweizerisches Pädiaterteam versandte an die Eltern von 256 Kindern, bei denen kürzlich eine Betalaktamallergie ausgeschlossen worden war, einen Fragebogen. 55,3\% der Eltern schickten eine Antwort zurück. Von diesen 141 Kindern waren 48 noch nicht wieder mit Betalaktamen behandelt worden. Von den übrigen 93, die in der Zwischenzeit wieder Betalaktame erhalten hatten, berichteten nur sieben von einer möglichen allergischen Reaktion - auf Amoxicillin alleine oder in Kombination mit Clavulansäure. Bei sechs von ihnen konnten ein Hautprick- test und ein oraler Provokationstest durchgeführt werden. Nur in einem Fall trat eine positive Reaktion auf, wie sich herausstellte allerdings nicht auf das zunächst verdächtigte Amoxicillin, sondern auf Clavulansäure. Zudem gab es eine Serumkrankheits-ähnliche Reaktion auf Cefaclor.

Rechnet man das Kind, das nicht weiter allergologisch untersucht werden konnte, den möglicherweise von einer Betalaktamallergie Betroffenen $\mathrm{zu}$, wiesen also nur zwei von $93 \mathrm{Kin}$ dern eine Überempfindlichkeit auf, nachdem dies bei ihnen zunächst aller- gologisch ausgeschlossen worden war. Bei den übrigen Kindern handelte es sich bei den verdächtigen Symptomen um solche der Infektionskrankheit und nicht um Reaktionen auf Betalaktame. Die Autoren folgern aus den Daten auch, dass die Gefahr einer Resensibilisierung durch Provokationstests eher gering ist.

Fazit: Wurde bereits einmal eine Betalaktamallergie ausgeschlossen, ist das Risiko, dass dennoch bei erneuter Antibiotikatherapie eine allergische Reaktion auftritt, gering. Nicht selten werden Symptome der behandelten Infektionskrankheit für eine Reaktion auf Betalaktame gehalten.

Ponvert C et al. Allergy to betalactam antibiotics in children: a prospective follow-up study in retreated children after negative responses in skin and challenge tests. Allergy 2007; 62: 42-6

\title{
Wenn der Magen auf die Lunge schlägt
}

\section{Der Zusammenhang zwischen Asthma bronchiale und gastroösopha- gealer Refluxkrankheit ist vielgestaltig. Im Rahmen einer klinischen Studie wurden nun die Prävalenz der gastroösophagealen Reflux- krankheit und zugleich das Ansprechen auf eine Therapie mit einem Protonenpumpeninhibitor bei Patienten mit schwer behandelbarem Asthma bronchiale untersucht.}

M alaiische Gastroenterologen suchten im Rahmen einer klinischen Studie bei insgesamt 30 Patienten mit schwer behandelbarem Asthma bronchiale nach dem parallelen Vorliegen einer Refluxkrankheit. Schwer behandelbares Asthma war definiert als anhaltende oder häufig rezidivierende Symptome trotz optimaler medikamentöser Therapie. Die Diagnostik der gastroösophagealen Refluxkrankheit beinhaltete neben der Anamnese auch die Gastroskopie und die 24-Stunden-pH-Messung.

Insgesamt fand sich bei 17 der 30 Patienten eine gastroösophageale Refluxkrankheit. Anschließend erhielten 27 der 30 Patienten - unabhängig da- von, ob zuvor eine Refluxkrankheit diagnostiziert worden war oder nicht -

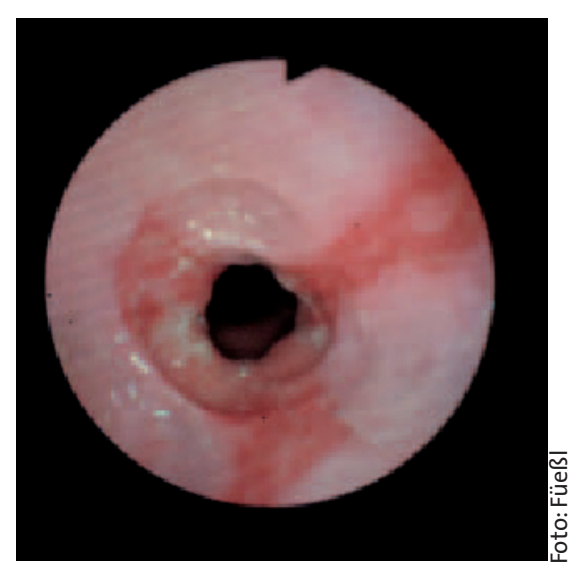

Refluxösophagitis Grad II über acht Wochen eine Therapie mit dem Protonenpumpenhemmer Lansoprazol in einer Dosierung von $30 \mathrm{mg}$ täglich. Danach wurde untersucht, ob sich die Asthmasymptomatik verbessert hatte.

Durch die Therapie konnte die pulmonale Symptomatik nur bei Patienten mit gleichzeitiger Refluxkrankheit günstig beeinflusst werden. Insgesamt berichteten zwölf von 16 Patienten mit Refluxkrankheit über eine deutliche Verbesserung der Asthmasymptome. Dagegen gab nur einer der elf Asthmapatienten ohne Refluxkrankheit eine leichte Verbesserung an.

Fazit: Die gleichzeitige Erkrankung an gastroösophagealem Reflux und schwerem Asthma bronchiale ist offenbar häufig. Die Behandlung der Refluxkrankheit mit einem Protonenpumpenhemmer verbessert auch die Asthmasymptomatik signifikant.

Wong $\mathrm{CH}$ et al. Gastro-oesophageal reflux disease in 'difficult-to-control' asthma: prevalence and response to treatment with acid suppressive therapy. Aliment Pharmacol Ther 2006; 23: 1321-7 